University of Wollongong

Research Online

Faculty of Engineering and Information

Faculty of Engineering and Information

Sciences - Papers: Part A

Sciences

$1-1-2014$

Dispatch strategy to minimise uncertainty in wind power generation in the Australian national electricity market

Amila Wickramasinghe

University of Wollongong, asw416@uowmail.edu.au

Lasantha G. Meegahapola

University of Wollongong, lasantha.meegahapola@rmit.edu.au

Ashish P. Agalgaonkar

University of Wollongong, ashish@uow.edu.au

Sarath Perera

University of Wollongong, sarath@uow.edu.au

Follow this and additional works at: https://ro.uow.edu.au/eispapers

Part of the Engineering Commons, and the Science and Technology Studies Commons

Research Online is the open access institutional repository for the University of Wollongong. For further information contact the UOW Library: research-pubs@uow.edu.au 


\title{
Dispatch strategy to minimise uncertainty in wind power generation in the Australian national electricity market
}

\author{
Abstract \\ 2014 ACPE. With increased penetration of wind power, scheduling generators to meet the forecast \\ demand of a power system is becoming an increasingly challenging task for the system operators. \\ Uncertainty associated with the generation expected from wind plants adds to the load demand \\ uncertainty, making it necessary to retain additional reserves to maintain the balance between demand \\ and supply of power. In the Australian national electricity market (NEM), sophisticated wind forecasting \\ techniques are employed to reduce the uncertainty in wind generation. Despite being able to project the \\ contribution of wind power to a reasonable accuracy, wind power plants are currently not considered to \\ be dispatchable resources in the NEM. This paper discusses the possibility of assigning firm generation \\ responsibilities to wind plants, especially in a dynamic electricity markets as the NEM, where the \\ generation scheduling happens between five to ten minutes ahead of actual dispatch.

\section{Keywords} \\ market, strategy, dispatch, minimise, uncertainty, wind, power, generation, australian, national, electricity \\ Disciplines \\ Engineering | Science and Technology Studies

\section{Publication Details} \\ A. Wickramasinghe, L. G. Meegahapola, A. P. Agalgaonkar \& S. Perera, "Dispatch strategy to minimise \\ uncertainty in wind power generation in the Australian national electricity market," in 2014 Australasian \\ Universities Power Engineering Conference, AUPEC 2014 - Proceedings, 2014, pp. 1-6.
}




\title{
Dispatch Strategy to Minimise Uncertainty in Wind Power Generation in the Australian National Electricity Market
}

\author{
Amila Wickramasinghe*, Lasantha Meegahapola, Ashish P. Agalgaonkar, Sarath Perera \\ Australian Power Quality and Reliability Centre, \\ School of Electrical, Computer and Telecommunications Engineering, \\ University of Wollongong, NSW 2522, Australia. \\ *Email: asw416@uowmail.edu.au
}

\begin{abstract}
With increased penetration of wind power, scheduling generators to meet the forecast demand of a power system is becoming an increasingly challenging task for the system operators. Uncertainty associated with the generation expected from wind plants adds to the load demand uncertainty, making it necessary to retain additional reserves to maintain the balance between demand and supply of power. In the Australian national electricity market (NEM), sophisticated wind forecasting techniques are employed to reduce the uncertainty in wind generation. Despite being able to project the contribution of wind power to a reasonable accuracy, wind power plants are currently not considered to be dispatchable resources in the NEM. This paper discusses the possibility of assigning firm generation responsibilities to wind plants, especially in a dynamic electricity markets as the NEM, where the generation scheduling happens between five to ten minutes ahead of actual dispatch.

Index Terms-Dispatchable, dispatch strategy, electricity markets, national electricity market, scheduled power plants, wind power.
\end{abstract}

\section{INTRODUCTION}

Scheduling power generators to meet the forecast demand is one of the key responsibilities of the power system operator. Since availability of generators cannot be fully guaranteed, additional generation resources need to be maintained as reserves, to be used if any scheduled generator becomes unavailable. Conventional thermal power plants exhibit certainty of generation and can deliver the required power when needed. Thus, they are identified as dispatchable power plants. Generators based on renewable resources such as wind and solar inherit the uncertainty of generation by virtue of their variable primary energy source. In particular, this uncertainty poses a significant barrier for wind power, restricting its widespread use even if grid parity is achieved through reduced wind power development costs. Given the importance of wind power as a non-polluting generation option, measures to overcome such barriers need to be examined.

Due to the advancements in climatology, development of high accuracy software models and accumulation of large amounts of data from increased number of measuring stations, wind speed forecasts are becoming increasingly accurate. As a result, generation from wind power plants have become more predictable, though still not to the extent required to consider them as reliable power sources. Thus, instead of assigning any capacity responsibilities, system operators tend to adjust the demand forecasts to accommodate the forecast wind generation.

Using certain control methods, it is possible for wind turbines to absorb part of the output power variations arising from fluctuating wind speeds. This allows wind farms to further improve their generation predictability. Since almost all modern wind turbines are able to regulate their blade pitch angles, it is possible to change the existing pitch control algorithms and include more grid friendly output power regulation schemes [1]. In variable speed wind turbines (VSWTs), controlling the rotor speed and changing the power transferred through the converters also allow output power regulation. Using these techniques, it is possible for wind turbines to curtail their output power and build an operating reserve, which can then be used to compensate for any reduction in the output caused by a drop in the wind speed [2]. Section II details these output regulating methods further.

Possibility of assigning greater responsibilities to wind generators, especially in the form of committed capacity, depends not only on the ability of these generators, but also on the way the power system is operated as a whole. Especially, if the power system is operated within an electricity market framework, the flexibility to treat wind power plants any different to other generators may not exist. Also, the timing of such assignments shall be within the time windows where accurate wind forecasting is possible. Based on the characteristics of the Australian national electricity market (NEM) presented in Section III, the suitability of Australian NEM to operate wind farms as scheduled power plants is deliberated in Section IV. Section IV also presents the steps that have to be taken by the wind farm operators as well as the system operator to enable scheduled operation of wind farms within the market rules, confirming the adaptability of the concept in the Australian NEM. A case study is presented in Section V, in which a wind plant successfully meets its capacity commitments while operating as a scheduled power plant. In comparison to discussions presented in other literature [3]-[5] on the possibility of maintaining a reserve capacity 
by wind turbines, this paper substantiates the applicability of such options in Australian NEM by proposing step-by-step operational guidelines in compliance with the market rules and validating these steps through a simulated case study. Section VI discusses the prospects of operating wind farms as scheduled power plants and the possibility of using the energy that could otherwise be wasted under fully scheduled operation.

\section{Regulation of Wind Turbine Power Output}

While it is generally accepted that a wind turbine cannot control its output and simply allow the output to vary according to the incident wind, such operation is observed only at wind speeds below the rated wind speed of the turbine. At wind speeds below the rated, the blade pitch angle $(\beta)$ is maintained at very low levels $\left(\beta<5^{\circ}\right)$ [6] to ensure maximum power extraction. However, when the incident wind speed is higher than the rated wind speed, $\beta$ is increased (up to $28^{\circ}$ ) to contain the electricity generation within the capacity rating of the generator and of any power electronic converters connected with the generator. Thus, at high wind speeds, the turbine control system maintains the electrical output steady at the rated generation level of the turbine.

\section{A. Output Regulation through Pitch Angle Control}

For a wind turbine with a rotor swept area of $A$, operating in a location with an air density of $\rho$, the amount of power that can be extracted from wind by the wind turbine $\left(P_{W T}\right)$ at a given wind speed $v$ can be given as;

$$
P_{W T}=\frac{1}{2} C_{p} \rho A v^{3}
$$

where $C_{p}$ is the power coefficient of the wind turbine rotor. The $C_{p}$ depends on the blade pitch angle $(\beta)$ and the tip speed ratio $(\lambda)$ of the turbine rotor.

The relationship between $\lambda, \beta$ and the $C_{p}$ can be presented as follows [7];

$$
C_{p}(\lambda, \beta)=\frac{1}{2}\left(\lambda-0.022 \beta^{2}-5.6\right) e^{-0.17 \lambda}
$$

As per (2), increase of $\beta$ causes $C_{p}$ to reduce, reducing the power output of the turbine. Therefore, wind turbines which have the capability to change their blade pitch angle, can build a capacity reserve by pitching the blades away from wind and curtailing their power outputs [3]. If the curtailed capacity is required to be supplied, the blade pitch angle can be adjusted back to the optimal value, enabling extraction of more power from wind. On the other hand, if further reduction of power output is needed, it can be easily achieved by increasing the blade pitch angle. Since the blade pitch mechanisms are relatively fast in modern wind turbines, the reserve capacities can be tapped in to at sufficiently fast rates. Also, with the precision controls wind turbines have for blade pitching, the power regulation can be maintained in a controlled manner, enabling tight regulation of the output amidst turbulent wind conditions.

\section{B. Output Regulation through Rotor Speed Control}

In variable speed wind turbines (VSWTs), in addition to pitch control, rotor speed control can also be used to run a wind turbine with a reduced load to maintain a reserve capacity [4].

At a given wind speed, the power that can be extracted by the wind turbine changes with the rotational speed of the wind turbine rotor. Furthermore, the rotor speed that corresponds to the maximum power extraction varies with the wind speed. Thus, in order to extract maximum power from wind, especially at low wind speeds, the speed of the rotor has to be altered according to the prevailing wind speed. This is the primary reason for the adoption of variable speed wind turbines as the preferred technology for wind generation over fixed speed wind turbines. Generally, variable speed wind turbines are operated at their optimal rotor speed [8] using the maximum power point tracking (MPPT) controllers, which controls the rotor speed based on the wind speed or the electrical power output from the generator.

The power coefficient $C_{p}$ in (1) can also be given given as follows [9];

$$
C_{p}(\lambda, \beta)=c_{1}\left(c_{2} \frac{1}{\Lambda}-c_{3} \beta-c_{4} \beta^{x}-c_{5}\right) e^{-c_{6} \frac{1}{\Lambda}}
$$

where,

$c_{1}-c_{6}$ are coefficients

$x$ is a turbine dependent parameter and $\Lambda$ is given by

$$
\frac{1}{\Lambda}=\frac{1}{\lambda+0.08 \beta}-\frac{0.035}{1+\beta^{3}}
$$

According to (3), $C_{p}$ changes with the tip speed ratio $(\lambda)$ of the turbine. $\lambda$ is defined as the speed of the rotor $(u)$ with reference to the wind speed $(v)$. Here, the tangential speed of the rotor $u$ can be expressed as $\omega R$ where $\omega$ is the angular velocity of the rotor and $R$ is the length of the blade. Therefore,

$$
\lambda=\frac{u}{v}=\frac{\omega R}{v}
$$

Based on (5), by changing the speed of the rotor $\omega$, the $C_{p}$ and consequentially, the power output can be changed. Generally, the rotor speed is selected to achieve the optimal $C_{p}$ which produces the maximum power for a given wind speed. However, if required, it is possible to operate the VSWTs at sub-optimal rotor speeds, reducing the real power output from the maximum possible at the given wind speed, indirectly creating a power reserve which can be tapped into, if a sudden increase in turbine power output is required. 


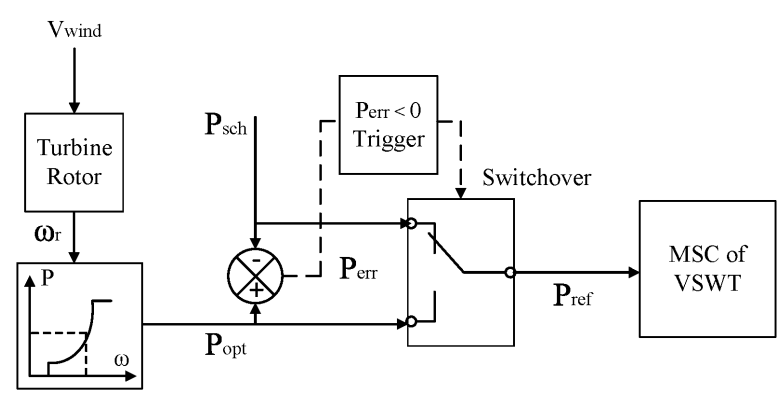

Fig. 1. Proposed control scheme for kinetic energy extraction

\section{Output Regulation through Rotor Kinetic Energy Extrac- tion}

It is also possible to provide a regulated power output from VSWTs for a short period, if the embedded kinetic energy in the turbine rotor is extracted to supplement the power supplied to the rotor by wind. In general, from the two back to back converters present in a VSWT, the converter coupled with the generator (called the machine side converter (MSC)) is responsible for the extraction of power from the generator [10]. If the MSC presents the generator of a VSWT with terminal conditions demanding a higher or lower power output than what the mechanical torque created by wind would allow, the generator would naturally comply with the power request by tapping into the kinetic energy available at the turbine rotor to meet the difference between the input and output power. This is similar to the inertial response of a synchronous generator directly coupled with an electrical network, which intrinsically taps into its kinetic energy-base to deliver the power demanded by the power system. Since the converters of a VSWT are typically configured to extract only the power available from wind, such kinetic energy extraction does not happen during normal operations, effectively restricting any inertial responses by VSWTs. However, if the power reference of the converter is intentionally set to a higher or lower value, the generator would use the rotor kinetic energy to fulfil its output commitment.

This method of output regulation was originally proposed [11] as a measure of emulating inertia, which VSWTs are not capable of providing, causing issues to system stability [12]. Changing the reference power level by a term proportional to $2 H \frac{d f}{d t}$ was proposed in [11], where $H$ is the inertia constant of the wind turbine. However, as presented in [13], it is possible to modify the converter power set point (i.e., the power extracted from the generator) entirely based on an external input.

By enabling the converters to take-in external power commands, either directly from the system controller, or through the wind farm operator, rotor kinetic energy can also be used to supplement the pitch and speed controlled output power regulation. Fig. 1 depicts the control method proposed for such output power regulation. Here, the power reference $\left(P_{\text {ref }}\right)$ given to the converter is altered to the scheduled power
$\left(P_{s c h}\right)$, if the maximum power that can be extracted from the turbine rotor $\left(P_{o p t}\right)$ corresponding to the prevailing wind speed $\left(V_{\text {wind }}\right)$ is less than what is scheduled $\left(P_{s c h}\right)$ for that dispatch interval. The difference $\left(P_{\text {err }}\right)$ between the scheduled power and the available power, defined by $P_{\text {err }}=P_{\text {opt }}-P_{\text {sch }}$ triggers the alteration of the power reference point as soon as it assumes a value less than zero (i.e., $P_{\text {sch }}>P_{\text {opt }}$ ).

This control scheme ensures delivery of the scheduled power, even if the power reserve maintained by the turbine through pitch/speed control is not adequate to meet the drop in wind speed. However, due to the limitation in kinetic energy available in the turbine rotor, regulation of power output through this method is possible only for a short period.

As a result of adjusting the output power, the speed of the turbine rotor changes, reflecting the change in kinetic energy embedded in the turbine. Here, unlike conventional synchronous generators equipped with governor controlled prime movers capable of bringing back the speed to the specified level, a wind turbine has to recover back to the optimal rotating speed by way of readjusting its output in a compensative manner. Thus, any regulated power output period has to be followed by a recovery phase, which will disrupt the normal operation of the wind turbine in the subsequent dispatch intervals. Thus, this method shall be used only as a back-up to the other two regulation methods.

\section{The Australian National Electricity Market}

The Australian NEM is the market mechanism for procurement and dispatch of electricity in the integrated electrical network across Australia's southern and eastern seaboard states. Covering the five states; Queensland, New South Wales, Victoria, South Australia and Tasmania, the NEM serves more than $80 \%$ of electricity consumers in Australia. Spanning over 5,000 km, the NEM has the longest ac transmission network in the world. The Australian energy market operator (AEMO) operates the NEM, performing the role of the system controller.

The NEM operates on an energy-only, day-ahead market with actual market clearing happening only five-minutes prior to each five minute dispatch interval. Market participants place bids on a gross energy pool to meet the forecast energy demand of the entire network for each dispatch interval. The forecast demand comprises the bids placed by large consumers and demand projections made by the AEMO, adjusted for the forecast wind generation based on AEMO's Australian wind energy forecasting system (AWEFS). AEMO schedules power generators to cater to the demand, based on a cost optimised selection process.

For one trading interval of 30 minutes (comprising 6 dispatch intervals), each generator unit can place ten different capacity bids under ten separate price bands (i.e., for the same 30 minute trading interval, a generator can offer parts of its available capacity at different prices). Furthermore, these capacity offers can be different for different trading intervals of the day. Thus, an energy bid of a generator unit would contain capacity bids under 10 price bands for the 48 trading intervals 
of the next day. Even though the bid gate closes everyday at 12.30 p.m., the actual dispatch cycle commences at 4.00 a.m. the next day, creating a wide gap between bidding and actual dispatch of the bid capacities. However, any changes required by the bidders to their offered capacities, can be notified to AEMO with reasons and the capacity offers can be adjusted without any alterations to price bands. The current bidding time frames require the generators to accurately estimate their generation capacities from 15.5 hours to 39.5 hours in advance, a requirement that makes it difficult for wind farms to engage in the bidding process.

In addition for energy, prices offered for frequency control ancillary services (FCAS) and market network services (MNS) as well as bids placed by dispatchable loads are considered in selecting the optimal dispatch schedule. Thus, the following objective function $\left(C_{T}\right)$ representing the total system cost is used to optimise the resources simultaneously;

$$
C_{T}=\begin{aligned}
& \text { Energy } \\
& \text { Cost }
\end{aligned}+\begin{aligned}
& \text { MNSP } \\
& \text { Cost }
\end{aligned}+\begin{aligned}
& \text { FCAS } \\
& \text { Cost }
\end{aligned}-\begin{aligned}
& \text { Load } \\
& \text { Revenue }
\end{aligned}
$$

AEMO runs its optimization program, the national electricity market dispatch engine (NEMDE), every five minutes to correspond to the next five minute dispatch interval and assigns the generators and network service providers (basically the HVDC interconnections) generation, reserve and power transfer capacities. At present, maintaining system voltage using network support and control ancillary services (NSCAS) is not managed through the market and is considered a responsibility of individual transmission system operators (TSOs).

\section{Proposed Approach FOR SCHEDUling Wind POWER IN THE NEM}

In order to minimise the uncertainty with the generation contribution from wind power plants, it is proposed to designate part of the available capacity of wind plants as firm/dispatchable capacity. Even though such a capacity commitment cannot be made over a long period, given the uncertainty in wind speeds, dynamic assignment of firm capacity would be possible and highly desirable for the power system. For such dynamic assignment of dispatchable capacity to be practicable, the scheduling of power plants needs to happen very close to actual dispatch. The Australian NEM, with its generator scheduling happening just before dispatch, and with no prior commitments or contracts with the generators to procure electricity, has the necessary framework to operate its wind power plants as scheduled generators and benefit from the minimised uncertainty of wind generation.

To treat a wind farm as a scheduled power plant in the Australian NEM, it shall essentially be able to offer firm capacities for the next trading day in accordance with the bidding process described in Section III. However, considering that at present, the demand projections are adjusted for forecast wind generation only five minutes ahead of dispatch, it is possible for the scheduling process to include last minute capacity bids from wind farms.
Inclusion of the firm generation capacities offered by wind farms does not change the objective function used in the dispatch optimisation program, as the schedulable wind farms can be treated in a similar manner to the conventional generators. However, it is likely that the constraints used to determine the FCAS requirement will now be changed with the increased certainty expected of the wind generation.

The following procedure is proposed for offering, accepting and dispatching wind power capacity in the NEM;

- Wind Farm Operator (5 to 10 minutes ahead of dispatch):

1) Forecast the wind speed profile for the dispatch interval for each wind turbine in the wind farm

2) Adjust the forecast wind profile for errors and uncertainties

3) Identify the minimum error-adjusted wind speed expected at each wind turbine

4) Using the power curves of each wind turbine, convert the minimum forecast wind speed to a forecast power output

5) Assign this capacity as the dispatchable capacity of the particular wind turbine

6) Arrive at the firm capacity available with the entire wind farm by aggregating individual dispatchable capacities

7) Offer the calculated firm capacity at the minimum price level to ensure clearance of the entire firm capacity available with the wind farm

- Market Operator (5 minutes ahead of dispatch):

1) Forecast the system demand without adjusting for the wind capacities which are offered as dispatchable capacities

2) Ensure the firm capacities offered by wind farms are cleared for dispatch

- Wind Farm Operator (during dispatch):

1) Upon receiving clearance for the offered firm capacity, set the output level of each turbine to the corresponding assigned capacity

2) If the actual wind speed incident on wind turbines drop below the forecast minimum speed, make up for the drop in power output of individual turbines by tapping in to the reserve capacities available with other turbines within the wind farm

3) Failing the above, ensure the scheduled output is provided by tapping into the kinetic energy-base of the wind turbines

4) Consider the recovery process from the kinetic energy extraction in offering capacity for subsequent dispatch intervals

- Market Operator (during dispatch):

1) In case wind farms fall short of delivering the scheduled capacities, engage the FCAS as appropriate

2) Use wind power as the primary source for 'regulation up' service, given the low marginal cost of generation of wind farms while operating as scheduled power plants. 


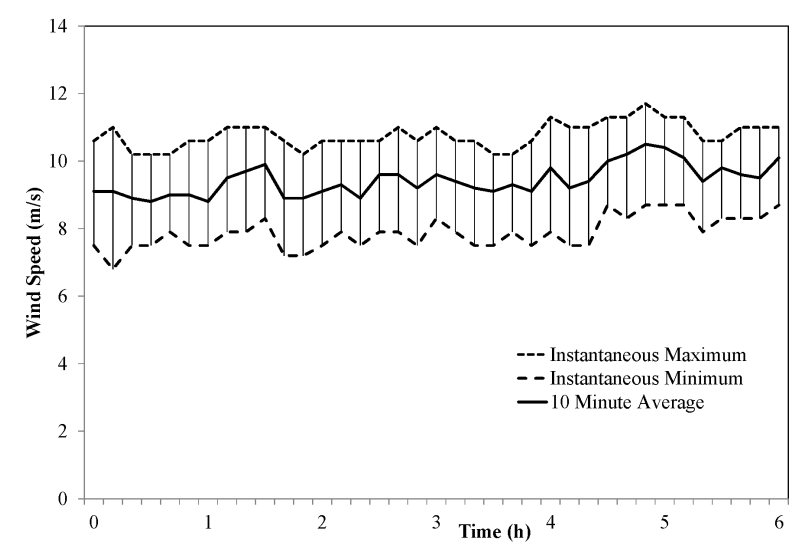

Fig. 2. Variations observed in wind speed within 10 minute intervals

\section{Case Study of a Wind Farm Operating as a SCHEDUlED POWER Plant}

In order to analyse the viability of scheduling wind farms to deliver firm capacity, a case study was carried out for a six hour period based on the wind farm operating scheme proposed in Section IV.

\section{A. Wind Speed Characteristics}

Wind speeds measured at a potential wind farm site was used for the case study. Instantaneous wind speed measurements were captured in the form of ten minute averages as well as instantaneous maximum and minimum over the averaged ten minute intervals. The standard deviation of wind speed over the averaged value was also recorded to represent the variation of wind speed within the ten minute averaging interval. The measurements were carried out at a height of $60 \mathrm{~m}$ and comparisons with simultaneous measurements at lower heights suggested negligible wind sheer, as generally expected for wind speeds at such heights at coastal wind sites as is the case of the measurement location. Thus, the recorded wind speeds were not adjusted for height in estimating power generation potential.

Analysis of instantaneous maximum and minimum wind speeds indicated a wide variation in wind speed, even within a ten minute interval. As per (1), the consequential variation of power generation can be much severe due to the cubic relationship the power generation $\left(P_{W T}\right)$ holds with wind speed $(v)$. However, due to the inertia of the turbine rotor, this variation is expected to be smoothened to a certain extent.

Fig. 2 emphasises one of the key challenges faced in scheduling wind turbines to deliver a fixed output. On top of any errors associated with forecasting wind speeds, the wind speed itself can change considerably within a five minute interval. Thus, it is essential for the turbine capacity scheduling to be based on the minimum expected wind speed and not on the average forecast.

In order to observe the variation of power output within five minute dispatch intervals, the ten minute average wind speeds were converted to a one minute wind speed profile using linear interpolation techniques. For the purpose of the case study, this interpolated one minute wind speed profile was used as the forecast wind speed profile. Furthermore, to study the effect of wind speeds varying within the maximum and minimum wind speed band, an 'actual' wind speed profile was synthesised, allowing the wind speed to assume random values within the maximum-minimum band of the dispatch interval in concern. The forecast wind speed against the synthesised pattern of the actual wind speed is shown in Fig. 3.

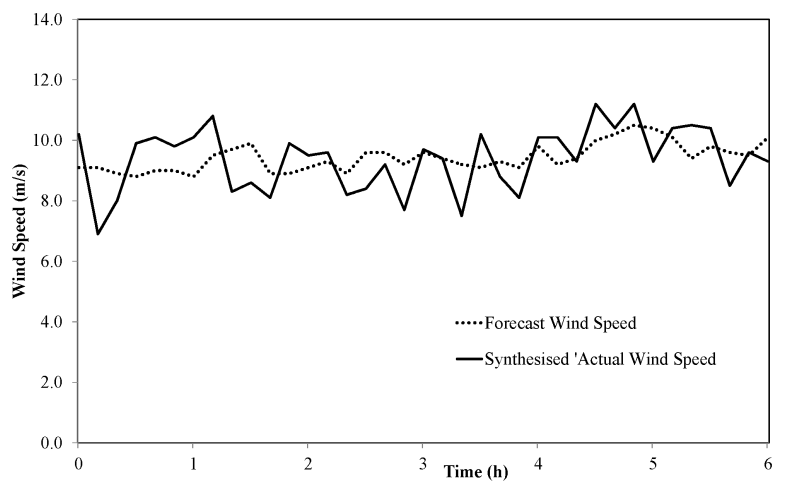

Fig. 3. Forecast and actual wind speeds used in the case study

\section{B. Wind Farm Characteristics}

The scheduled operation of wind power was tested on a wind farm comprising 40 full converter (Type D) wind turbine generators, each of 2.5 MW capacity. Since the focus of the study is to verify the technical feasibility of accommodating wind speed variations when wind farms are operated as scheduled power plants, the power system simulation was carried out using a single machine infinite bus (SMIB) configuration. DIgSILENT Power Factory was used as a software tool to implement the proposed control schemes and to observe the behaviour of the wind farm whilst in operation as a scheduled power plant.

In carrying out the case study, the capacity scheduling scheme presented in Fig. 4 was adopted. A 20\% error factor was used in adjusting the forecast wind speed profile to establish the minimum wind speed expected at each dispatch interval.

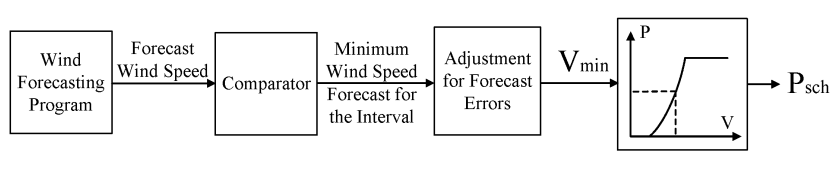

Fig. 4. Scheme used for scheduling of wind turbines

\section{Results AND Discussion}

Power output from the wind farm over the analysed six hour period is presented in Fig. 5, along with the estimated maximum power that was available at the wind farm during the same period. It is noted that, amidst the variation in wind speed experienced by the wind farm, it had managed to dispatch its 
scheduled output for the entire duration of the study, validating that such an operation is feasible.

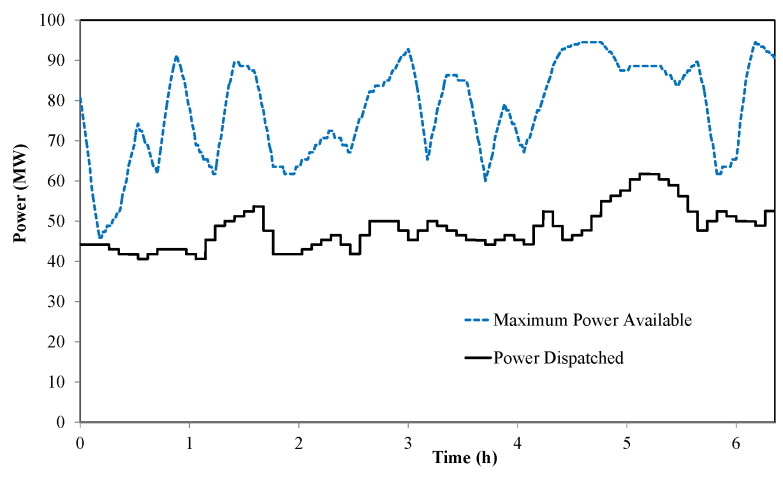

Fig. 5. Wind farm output under scheduled operation

Despite the positive results observed in the case study, it is still likely that the ability of a wind farm to honour its capacity commitments could be compromised if the error in the wind forecast is higher. However, with better understanding of forecasting errors and variability of wind speeds at different geographic locations, wind developers would be able to offer a considerable portion of their estimated generation as firm capacity, increasing the value the energy supplied by these wind farms.

It is also noted that, $38 \%$ of the energy available at the wind farm was 'spilled' (not dispatched) (Fig. 5). That is, in the process of minimising the uncertainty in generation, the wind farm has incurred a considerable loss in generation. To overcome this issue, it is possible to allow the wind farms to produce the maximum possible output and value the supplied energy at different rates, based on capacities offered as dispatchable and non-dispatchable. This way, the wind farms could continue to operate in the same manner as they are operated at present, but with the exception of dispatching part of its generation capacity through the energy market, considerably reducing the uncertainty attributed to wind generation.

If wind power penetration levels become too high or the electricity markets become fully competitive, operation of wind farms as scheduled power plants could become a mandatory requirement. Under such circumstances, wind farms would be compelled to 'spill' part of their generation to ensure a steady output. It is possible to use this unutilsed capacity to provide ancillary services such as frequency regulation services and reactive power supply. The viability of those options would depend on the cost of wind power development and the prices offered for such ancillary services.

\section{CONCLUSION}

This paper discussed a strategy to dispatch wind farms as scheduled power plants in order to minimise the uncertainty brought about by wind generators to power system operations. Implementation of the proposed scheduling strategy within the framework of the Australian NEM was discussed, proposing minimal changes to the current method of scheduling generators. Using a case study carried out on a $100 \mathrm{MW}$ wind farm, it was demonstrated that by adopting the proposed strategy, it is possible to meet capacity commitments made by wind generators amidst fluctuating wind conditions. The possibility of adding value to the energy generated by wind power plants was discussed, highlighting the alternating uses of capacity that could otherwise go waste, if wind farms are to guarantee their generation.

\section{REFERENCES}

[1] T. Senjyu, R. Sakamoto, N. Urasaki, T. Funabashi, H. Fujita, and H. Sekine, "Output power leveling of wind turbine generator for all operating regions by pitch angle control," IEEE Trans. Energy Convers., vol. 21, pp. 467-475, June 2006.

[2] P. Venne and X. Guillaud, "Impact of wind turbine controller strategy on deloaded operation," in Integration of Wide-Scale Renewable Resources Into the Power Delivery System, 2009 CIGRE/IEEE PES Joint Symposium, July 2009.

[3] L. Holdsworth, J. B. Ekanayake, and N. Jenkins, "Power system frequency response from fixed speed and doubly fed induction generatorbased wind turbines," Wind Energy, vol. 7, no. 1, pp. 21-35, 2004.

[4] R. de Almeida, E. Castronuovo, and J. Peas Lopes, "Optimum generation control in wind parks when carrying out system operator requests," IEEE Trans. Power Syst., vol. 21, no. 2, pp. 718-725, 2006.

[5] G. Ramtharan, J. Ekanayake, and N. Jenkins, "Frequency support from doubly fed induction generator wind turbines," IET Renewable Power Generation, vol. 1, pp. 3-9, Mar. 2007.

[6] Z. Lubosny, Wind Turbine Operation in Electric Power Systems: Advanced Modeling. Engineering online library, Springer, 2010.

[7] O. Wasynczuk, D. T. Man, and J. P. Sullivan, "Dynamic behavior of a class of wind turbine generators during random wind fluctuations," IEEE Transactions on Power Apparatus and Systems, vol. PAS-100, pp. 2837 2845, June 1981.

[8] I. Cadirci and M. Ermis, "Double-output induction generator operating at subsynchronous and supersynchronous speeds: steady-state performance optimisation and wind-energy recovery," IEE Proceedings on Electric Power Applications, vol. 139, no. 5, pp. 429-442, 1992.

[9] R. Nijssen, M. Zaaijer, W. Bierbooms, G. Van Kuik, D. Van Delft, and T. van Holten, "The application of scaling rules in up-scaling and marinisation of a wind turbine," in European Wind Energy Conference and Exhibition (EWEC), 2001.

[10] R. Pena, J. Clare, and G. Asher, "Doubly fed induction generator using back-to-back PWM converters and its application to variable-speed windenergy generation," IEE Proceedings on Electric Power Applications, vol. 143, no. 3, pp. 231-241, 1996.

[11] J. Ekanayake, L. Holdsworth, and N. Jenkins, "Control of DFIG wind turbines," Power Engineer, vol. 17, pp. 28-32, Feb. 2003.

[12] L. Meegahapola and D. Flynn, "Impact on transient and frequency stability for a power system at very high wind penetration," in Proc. IEEE Power Eng. Soc. General Meeting, Jul. 2010.

[13] N. Ullah, T. Thiringer, and D. Karlsson, "Temporary primary frequency control support by variable speed wind turbines: Potential and applications," IEEE Trans. Power Syst., vol. 23, pp. 601-612, May 2008. 